\title{
Alteraciones bioquímicas de los lípidos en los alimentos vegetales. I. Formación de los hidroperóxidos lipídicos.
}

\author{
Por R. Zamora, F. J. Hidalgo y M. Alaiz \\ Instituto de la Grasa y sus Derivados. Avenida Padre García Tejero, 4. \\ 41012 Sevilla
}

RESUMEN

Alteraciones bioquímicas de los lípidos en los alimentos vegetales. I. Formación de los hidroperóxidos lipídicos.

En este trabajo se hace una revisión de los enzimas responsables de la degradación enzimática de los lipidos en los alimentos vegetales. Esta primera parte describe la formación de los hidroperóxidos lipidicos producida por la acción secuencial de lipasas y lipoxigenasas.

PALABRAS-CLAVE: Alimento vegetal - Información (articulo) Lipasa - Lipoxigenasa - Oxidación lipldica.

\section{SUMMARY}

Biochemical changes of lipids in plant foods and feeds. I. Lipid hydroperoxides formation.

The enzymes of the lipoxygenase pathway in plant foods are reviewed. This part analyzes the enzymes related to the formation of the lipidic hydroperoxides. Lipases and lipoxygenases in plant foods are described.

KEY-WORDS: Information (paper) - Lipase - Lipid oxidation - Lipoxygenase - Plant food.

\section{INTRODUCCION}

Durante su procesado y/o almacenamiento, los alimentos están sujetos a una serie de alteraciones que tienen una importante repercusión en la calidad de los mismos. Entre estas alteraciones, la oxidación lipídica ocupa un lugar primordial a causa de los cambios que produce en los alimentos, con consecuencias que implican modificaciones en su aroma, su sabor, la textura, la consistencia y la apariencia, así como su valor nutritivo y su seguridad.

Tanto las plantas como los animales poseen sistemas bioquímicos que transforman secuencialmente los glicerolípidos en derivados de los ácidos grasos a través de una serie de pasos que son conocidos generalmente como cascada de los ácidos grasos poliinsaturados. Aunque se dan algunas similitudes entre las cascadas animal y vegetal, entre ambas existen sustanciales diferencias no sólo en las rutas que siguen sino también tanto en los mecanismos de control que regulan el proceso como en los productos finales for- mados. Típicamente la cascada consiste en una hidrólisis de los glicerolípidos presentes por enzimas lipolíticos, seguida de una oxidación de los ácidos grasos liberados y termina con una transformación o descomposición enzimática de los hidroperóxidos o endoperóxidos producidos en el paso de oxidación. Los compuestos producidos en la cascada usualmente tienen un papel indispensable en el control de ciertas funciones metabólicas del organismo (1).

En los mamíferos, la cascada del ácido araquidónico ha sido, y lo sigue siendo en la actualidad, objeto de intensas investigaciones a causa de la importancia biomédica de los productos resultantes (2-4). Un daño al tejido desencadena el inicio de la cascada. El ácido araquidónico, liberado de los fosfolípidos por acción de la fosfolipasa $A_{2}$, puede ser oxidado por la lipoxigenasa. Este enzima, cuya existencia en animales ha sido cuestionada hasta hace pocos años (5), $y$ que hoy en dia ha sido encontrado principalmente en plaquetas (6) y leucocitos (7), produce la formación de hidroperóxidos en el ácido araquidónico. La intervención posterior de otros sistemas enzimáticos convierten estos hidroperóxidos en ácidos hidroxitetraénicos, epoxihidroxitriénicos y trihidroxitriénicos, y leucotrienos. Por otra parte, el ácido araquidónico también puede ser oxidado por la prostaglandinendoperóxido-sintetasa a la prostaglandina $G_{2}$ que puede a su vez sufrir sucesivas transformaciones $y$ formar otro conjunto de compuestos como son tromboxanos, prostaglandinas y prostaciclinas. Los distintos productos formados en estas rutas tienen un papel primordial en la agregación de plaquetas, inflamación, quimiotaxis y contracción del músculo liso, y se ha sugerido que algunos de estos compuestos podrian estar relacionados con enfermedades coronarias (8).

Los vegetales, por su parte, responden de manera algo distinta al daño al tejido. Debido a que los principales ácidos grasos que poseen son los ácidos linoleico y linolénico, éstos son los que sustituyen al araquidónico en la cascada cuyas rutas conocidas se han recogido en la Figura 1. Los ácidos grasos poliinsaturados son liberados de los glicéridos, glicolípi- 
dos (principalmente galactolípidos) y fosfolípidos por acción de las lipasas e hidrolasas. La oxidación posterior de estos ácidos grasos por la lipoxigenasa produce los correspondientes hidroperóxidos que a su vez son convertidos por diferentes enzimas en una mezcla compleja de compuestos. A diferencia de la los alimentos vegetales no procesados almacenados congelados durante largos períodos.

El objetivo de esta revisión es la discusión del estado actual del conocimiento de los distintos sistemas enzimáticos involucrados en estos procesos de oxidación lipídica en alimentos vegetales, describien-

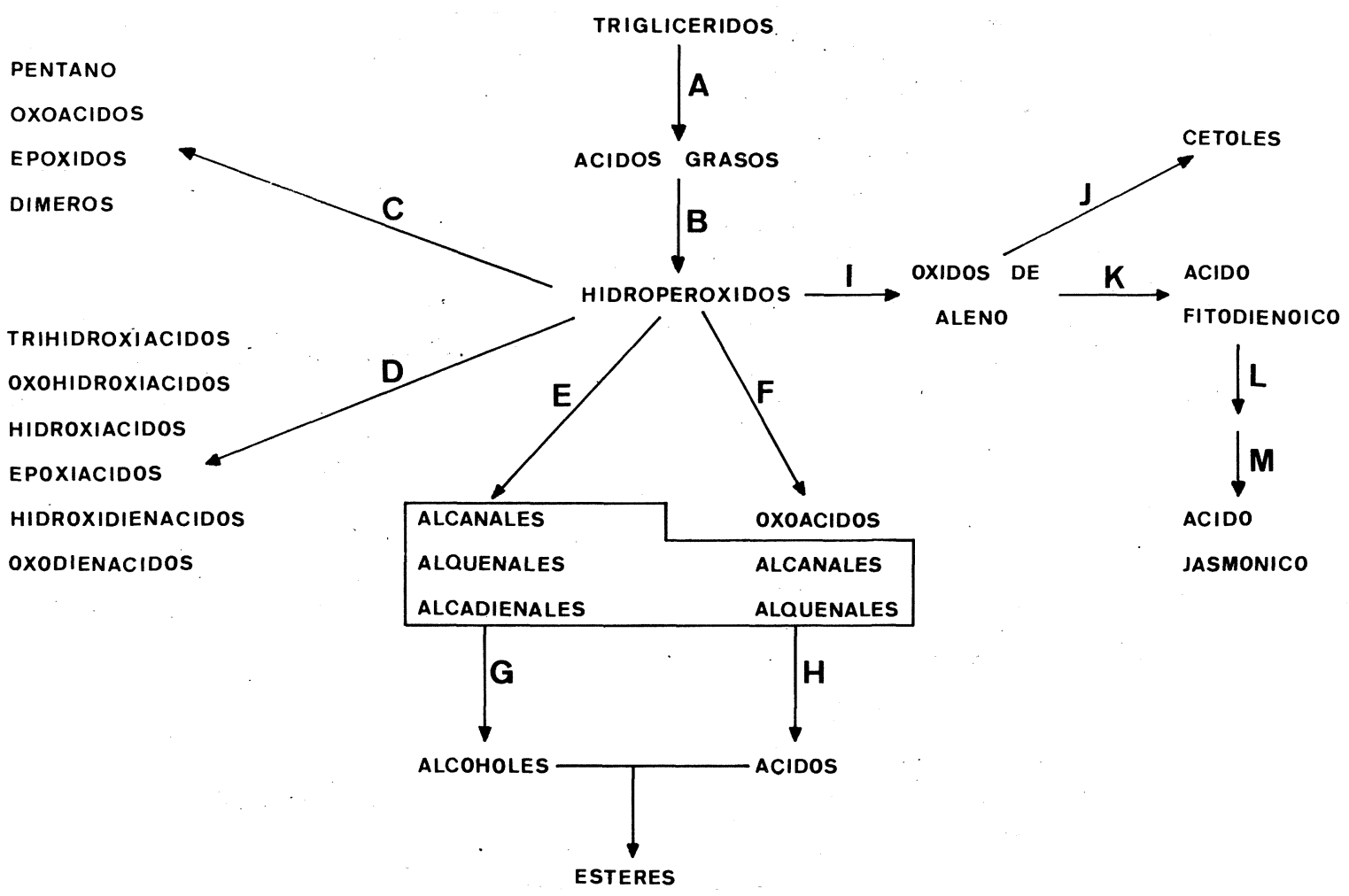

Figura 1

Transformaciones bioquímicas conocidas que sufren los lipidos en los alimentos, habitualmente llamadas ruta de la lipoxigenasa o cascada de los ácidos linoleico y linolénico. Enzimas que intervienen: a, lipasas; b, lipoxigenasa; c, lipoxigenasa anaeróbica (catálisis seudoenzimática); d, lipoxigenasa aeróbica (catálisis seudoenzimática); e, lipoxigenasa (catálisis seudoenzimática); $f$, hidroperóxido liasa; $g$, alcohol deshidrogenasa; $h$, aldehído deshidrogenasa; i, hidroperóxido dehidrasa; j, hidrólisis (no enzimática); k, óxido de aleno ciclasa; l, ácido cetofitodienoico reductasa; $m$, reacciones de B-oxidación.

cascada del ácido araquidónico el significado fisiológico de esta cascada es mucho menos conocido aunque recientes investigaciones parecen apuntar que puede estar implicada en procesos de daño a tejidos (9), análogamente a lo que ocurre con la cascada de los mamíferos, y en procesos de senescencia (10).

La consecuencia más evidente de esta cascada en vegetales es el desarrollo de olores y sabores. Generalmente la trituración, congelación, maceración o procesado dé los alimentos provoca un daño a sus tejidos desencadenando toda la serie de transformaciones enzimáticas que apuntábamos anteriormente. El resultado es el olor a verde de las hojas trituradas, el característico olor del pepino cuando se corta, las notas frescas de las frutas y verduras, 0 , por el contrario, los olores desagradables que desarrollan do lo que hoy en día ya empieza a ser conocido como la cascada de los ácidos linoléico y linolénico.

Durante mucho tiempo se ha sabido que la oxidación de los lípidos insaturados en los alimentos produce compuestos aldehídicos volátiles que, junto con los alcoholes derivados de su reducción, son los responsables de los olores desagradables que desarrollan los alimentos durante su procesado o almacenaje. Debido a esto y como es sabido que la lipoxigenasa es la responsable principal del proceso, muchos vegetales, como las judías y los guisantes, son hervidos brevemente antes de su envasado o congelación para desactivar la lipoxigenasa al mismo tiempo que otras enzimas. Sin embargo, estos tratamientos no conllevan sólo ventajas $y$, en algunos casos, la desactivación de la lipoxigenasa produce cambios en la textura 
y en otros atributos que contribuyen a la calidad del alimento.

El flavor de un alimento es la suma de los efectos de distintos compuestos a diferentes concentraciones. Así, por ejemplo, el nonenal y nonadienal y sus correspondientes alcoholes imparten el característico flavor del pepino o del melón; y en el té, trazas de hexanal y hexenal son deseables. Por el contrario estos dos mismos compuestos, hexanal y hexenal, pueden ser considerados desagradables en la soja debido a que le confiere notas "verdes" o de "pasto". Así la diferencia entre un flavor agradable y otro desagradable muchas veces estriba tan sólo en las concentraciones del mismo y/o en un equilibrio entre los niveles relativos de los distintos flavors.

Las isomerizaciones y deshidrogenaciones de los aldehidos formados también tiene una considerable influencia en el flavor de los alimentos vegetales. Así, por ejemplo, la transformación cis-trans de un doble enlace puede cambiar el límite de detección de un flavor de tal manera que un aroma potente sea de menor importancia o viceversa. El Z-4-heptenal, que fue encontrado como producto de oxidación en el bacalao, tiene un límite de detección de $0,04 \mathrm{ppb}$ en agua y 0.5-1.6 ppb en aceite. Su transformación en el isómero trans sube los límites de detección unas 200 veces (11). Otro ejemplo en este sentido pero ilustrando esta vez la importancia de un doble enlace puede ser la comparación entre la cis-1,5-octadien-3ona y la 1-octen-3-ona. La primera, un producto de la oxidación de los ácidos n-3 pentainsaturados que ha sido encontrada en la mantequilla, es detectada a una concentración 100 veces mayor que la segunda, un producto de la oxidación de los ácidos n-6 pentainsaturados (12).

Todos estos ejemplos ilustran la importancia que pequeños cambios en la proporción y estructura de los compuestos producidos pueden tener en la aceptabilidad de los alimentos. En esta revisión se discutirá cómo los distintos productos son originados y cuáles son los mecanismos conocidos de control de la ruta.

\section{LIBERACIÓN DE LOS ÁCIDOS GRASOS: ENZIMAS LIPOLÍTICOS.}

Todo el proceso se inicia con la lipolisis de lípidos complejos produciendo la liberación de los ácidos grasos. Este proceso, que ocurre después de la maduración o de que se ha producido un daño al tejido, es llevado a cabo por enzimas lipolíticos provocando lo que a menudo ha sido llamado rancidez hidrolitica.

Hay toda una variedad de enzimas lipolíticos conocidos, los cuales a menudo son específicos en su capacidad de hidrolizar un enlace acil-glicérido de un sustrato lipídico definido (13). Estos enzimas son usualmente considerados hidrolasas cuando usan el agua como sustrato. Sin embargo, debe ser tenido en cuenta que la mayoría de las hidrolasas pueden actuar como transferasas cuando el cosustrato es un alcohol. Este punto no será discutido en este trabajo.

Generalmente cada lipasa hidroliza especificamente un enlace acil-glicérido de un sustrato lipídico definido. De acuerdo con esto se clasifican en lipasas verdaderas, que hidrolizan triglicéridos únicamente, y acilhidrolasas. Entre estas últimas merece señalar a las fosfolipasas, que actúan sobre los fosfolípidos y las glicosildiglicéridolipasas que hidrolizan glicolípidos.

Las lipasas verdaderas se encuentran asociadas a la membrana de los oleosomas o cuerpos lipídicos. Son relativamente específicas para los triglicéridos que contienen los ácidos grasos mayoritarios en esas especies. Asi, por ejemplo, se ha encontrado que la lipasa del maíz es más activa con los triglicéridos conteniendo los ácidos oléico y linoleico que son los ácidos grasos mayoritarios en el maíz. Por su parte, la lipasa de la semilla de olmo, por ejemplo, que contiene altos porcentajes del ácido caproico, es casi específica de la tricaproina (14). Esta especificidad hacia ciertos ácidos grasos puede también ser observada en diacil y monoacil-gliceroles, aunque se va perdiendo con la liberación de ácidos grasos (15). Esta pérdida gradual en la especificidad de las lipasas pudiera tener una importancia fisiológica. Cada molécula de triglicérido no está sólo compuesta del ácido graso mayoritario sino de uno mayoritario y otros minoritarios. Así, después de que la lipasa ha hidrolizado el ácido graso mayoritario, ésta pierde especificidad y puede hidrolizar los restantes ácidos grasos (13).

En general, todas las lipasas verdaderas no tienen actividad en semillas no germinadas y su actividad se incrementa durante la germinación y postgerminación disminuyendo posteriormente cuando desaparecen los triglicéridos. Esto puede tener interés en las semillas de alfalfa germinadas que se han popularizado enormemente en países como los EE.UU. y otros.

Para las semillas de ricino, y en general para las lipasas verdaderas, se ha propuesto (16) un mecanismo de acción en él que, y a diferencia del mecanismo propuesto para la lipasa pancreática, los tres grupos éster del glicerolípido son hidrolizados. Este proceso ocurre mayoritariamente durante la germinación donde el $90 \%$ de la reserva lipídica de la planta, que son triricinolinoleilgliceroles, es completamente degradado a ácidos grasos que son posteriormente utilizados (17). Estudios realizados por Noma y Borgstrom demostraron que la secuencia de la reacción es triacilglicerol que pasa a 1,2-diacilglicerol, posteriormente a 1-y 2-monoacilglicerol y finalmente a glicerol (16). 


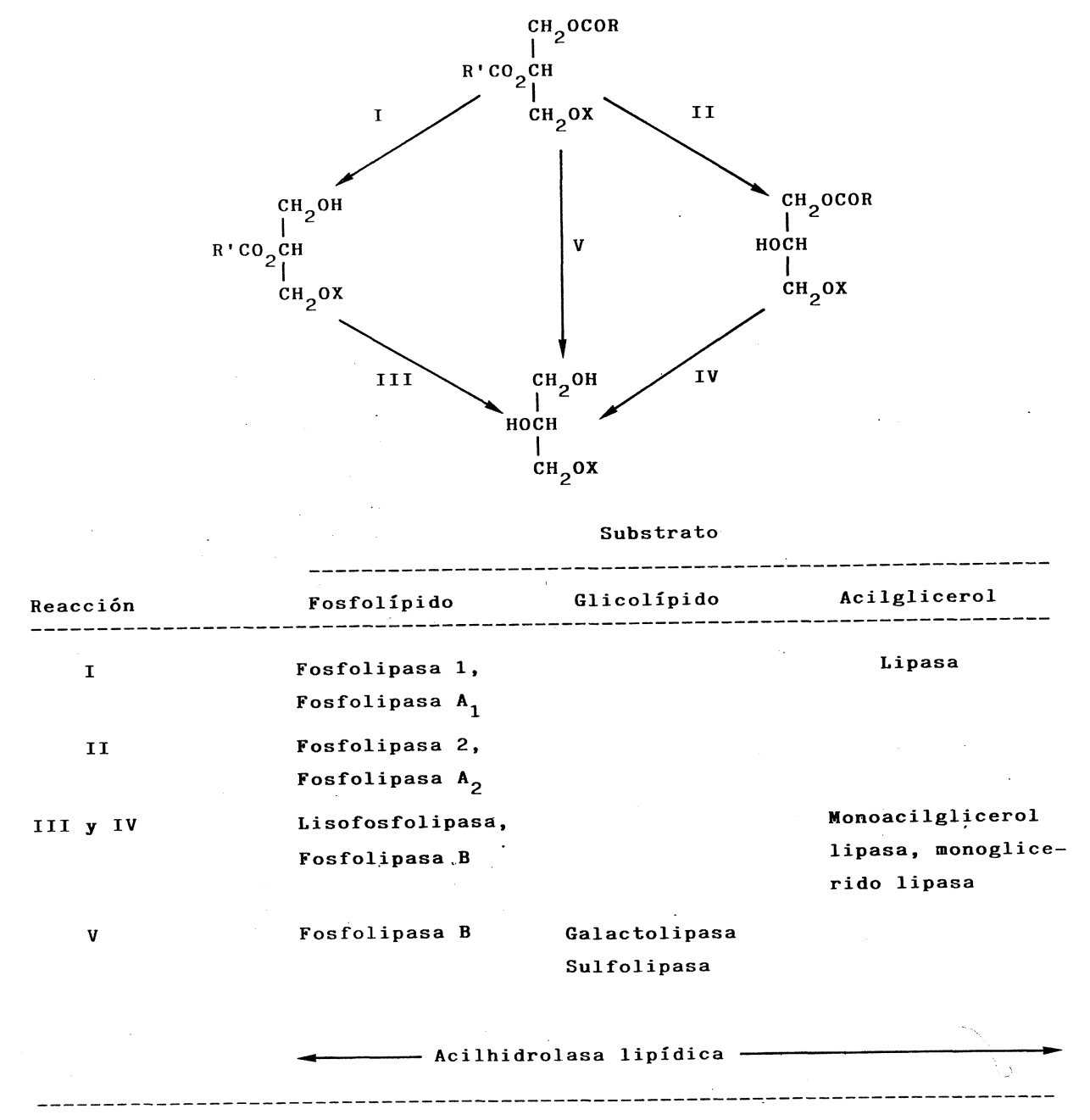

Figura 2

Mecanismo de acción de las acilhidrolasas y nombres que reciben de acuerdo al paso en el que actúan.

Dentro de este grupo de lipasas verdaderas se han encontrado unas lipasas que son solubles porque no están asociadas a membranas. Aún no han podido ser purificadas y el conocimiento existente sobre las mismas es muy escaso (15).

Las otras lipasas, las acilhidrolasas, entre las que se encuentran las fosfolipasas, glicolipasas y un grupo de lipasas no específicas, pueden ser agrupadas en un único grupo de enzimas que desacilan glicerolípidos del tipo de la figura donde $R$ y $R^{\prime}$ pueden ser $H$ o un grupo acilo y $X$ puede ser $H$ (acilgliceroles), un éster fosfato (fosfolípidos) o un residuo glicolípido (galactolípidos o sulfolípidos). Para estas lipasas, se ha propuesto la ruta que se recoge en la Figura 2. Dependiendo de la reacción en que actúen estos enzimas reciben diferentes nombres que son los que se recogen en la parte inferior de la figura.

A diferencia de las lipasas verdaderas, las acilhidrolasas no son especificas. Así, por ejemplo, una acilhidrolasa aislada de la patata por Galliard (18) hidroliza muy bien sustratos tan diferentes como galactolípidos, fosfolípidos y monooleina. Por su parte, otra acilhidrolasa aislada también de la patata por Hirayana et al, (19) mostró una alta afinidad por galactolípidos, fosfolípidos y acilglucósidos. El conocimiento de otras acilhidrolasas es aún muy limitado.

La maceración, la molienda y la homogeneización de tejidos normalmente desencadena una rápida lipolisis ya que los enzimas lipolíticos son puestos en contacto con los sustratos lipídicos. De esta manera tanto los triglicéridos de reserva como los lípidos de las membranas pueden ser hidrolizados. Esto podría explicar la lipolisis que frecuentemente se produce después de la maceración o molienda de las semillas oleaginosas o la rápida producción de volátiles procedentes de la degradación lipídica que sigue a la homogeneización.

La lipolisis también puede ocurrir en sustratos "en 
seco" tal como ocurre en los copos de avena que tan asiduamente están siendo empleados para los desayunos (20). En estas sustancias secas el agua necesaria para la hidrólisis es suministrada por equilibrio con la humedad del aire.

\section{OXIDACIÓN DE LOS ÁCIDOS GRASOS: LIPOXIGENASAS}

Una vez que los ácidos grasos son liberados por enzimas lipolíticos se puede producir la oxidación de los ácidos grasos poliinsaturados por la lipoxigenasa. Las lipoxigenasas, tanto en plantas como en animales, son dioxigenasas de ácidos grasos poliinsaturados. Normalmente se considera que el término genérico "lipoxigenasa" agrupa a un conjunto de enzimas que catalizan la oxidación de lípidos conteniendo un sistema Z,Z-1,4-pentadieno para producir los correspondientes hidroperóxidos conjugados. Los sustratos naturales de este enzima en plantas son, por tanto, los ácidos linoleico y linolénico. Sin embargo, recientes hechos encontrados parecen poner en duda que la condición exclusiva para la actuación de las lipoxigenasas sea el sistema Z,Z-1,4-pentadieno. Por una parte se ha observado que ácidos grasos de 8 a 12 átomos de carbono, aún teniendo el sistema antes mencionado, no actúan como sustratos de la lipoxi- genasa (21). Por otra, se ha comprobado que todos los $C_{18}$ que pueden actuar como sustratos tienen el sistema pentadieno en los carbonos 9 y 12 de la cadena (21). Isómeros geométricos del ácido linoléico no dan la reacción y sus isómeros posicionales pueden actuar como sustratos para la lipoxigenasa de hojas de té pero su actividad hacia ellos es menos de la mitad que la encontrada para el ácido linoleico (21). Otros derivados del ácido linoleico como sus ésteres metílicos, su alcohol y su aldehido actúan solamente a $\mathrm{pH}$ 4.5 y en menor extensión (21). De todo lo dicho se puede concluir que el sistema Z,Z-1,4-pentadieno debe estar localizado en las posiciones $\omega-6$ y $\omega-9$ y que la presencia del grupo carboxilo es importante pero no esencial.

La lipoxigenasa está ampliamente distribuida en plantas. Durante un tiempo se pensó que estaba restringida a legumbres y poco más, pero ya en el año 1971 Pinsky et al. (32) encontraron actividad lipoxigenasa en más de 40 especies vegetales. Entre las especies que presentan una mayor actividad lipoxigenasa destacan la patata, la berenjena, la soja, las alcachofas, los guisantes, la coliflor, al aguacate, el tomate y la lechuga.

A nivel subcelular, actividad de lipoxigenasa ha sido encontrada en diferentes fracciones dependiendo particularmente de la planta usada. Asi en las hojas

Tabla 1

Principales características de las isoenzimas de la lipoxigenasa de soja*

\begin{tabular}{|c|c|c|c|}
\hline & L-1 & $\mathrm{L}-2$ & $\mathbf{L}-\mathbf{3}$ \\
\hline Peso molecular & 102,000 & 100,000 & 100,000 \\
\hline Punto isoeléctrico & 5.68 & 6.25 & 6.15 \\
\hline pH óptimo & $9.0-9.5$ & $6-7$ & $6-7$ \\
\hline $\mathrm{Km}$ aprox. con ácido linoleico $(\mu \mathrm{M})$ & 12 & 16 & 340 \\
\hline Oxidación (moles ácido linoleico/min/mol) & 15,800 & 6,500 & -- \\
\hline $\begin{array}{l}\text { Estabilidad frente al calor (vida media } \\
\text { a } 69^{\circ} \mathrm{C} \text { en min.) }\end{array}$ & 25 & 0.7 & -- \\
\hline $\begin{array}{l}\text { Especificidad de sustrato } \\
\qquad(\text { referido al ácido linoleico) }\end{array}$ & & & \\
\hline Linoleato de metilo & 0.10 & 0.52 & 2.0 \\
\hline Metano sulfonato de linoleilo & 0.17 & 1.80 & 1.75 \\
\hline Acetato de linoleilo & 0.06 & 0.39 & 0.75 \\
\hline Acido araquidónico & 1 & 1.5 & 0.5 \\
\hline
\end{tabular}

Extraido de ref. 27 y 43 . 


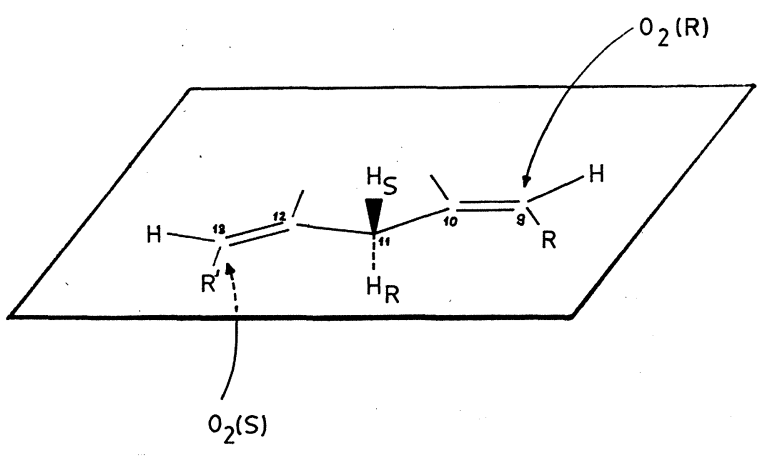

Figura 3

Estereoquímica del mecanismo de acción de la lipoxigenasa. se acumula en los cloroplastos (23), en la alfalfa ha sido encontrada tanto en cloroplastos como en mitocondrias (24), en patatas, guisantes y pepino ha sido encontrada en la fracción microsomal (25) y, finalmente, en flores de coliflor ha sido encontrada en una fracción de densidad similar a los plástidos aunque diferentes a éstos (25). El grupo de Galliard ha puesto de manifiesto la confusión que existe sobre el tema en la bibliografía, confusión que, por otra parte, ha sido alentada por la creencia ampliamente extendida de que la lipoxigenasa es un enzima citoplasmático. Hoy en día parece ser que ella pudiera estar localizada en orgánulos muy frágiles (26).

En la actualidad se conocen distintos isoenzimas de la lipoxigenasa. En semillas de soja, donde la

Tabla II

Especificidad de oxidación de diversas lipoxigenasas.

\begin{tabular}{|c|c|c|c|c|c|}
\hline Fuente & Tejido & $\begin{array}{l}\text { Acido } \\
\text { geaso }\end{array}$ & $\begin{array}{l}\text { Hidroperóxido producido } \\
\text { 9-:13- (\%) }\end{array}$ & pH & Referencia \\
\hline Alfalfa & Semilla & LH & $50: 50$ & 6.8 & 30 \\
\hline Altramuz & Semilla & $\mathrm{LH}$ & $48: 52$ & - & 31 \\
\hline Arroz & Germen & LH & $97: 3$ & 7.0 & 32 \\
\hline Garbanzo & Semilla & LH & $45: 55$ & - & 31 \\
\hline Guisante & Semilla & $\mathrm{LH}$ & $58: 42$ & 6.3 & 33 \\
\hline Lenteja & Semilla & LH & $26: 74$ & - & 31 \\
\hline \multirow[t]{2}{*}{ Lino } & Semilla & $\mathrm{LH}$ & $20: 80$ & 6.5 & 34 \\
\hline & & $\operatorname{LnH}$ & $12: 88$ & 665 & 34 \\
\hline \multirow[t]{2}{*}{ Maiz } & Germen & $\mathrm{LH}^{\circ}$ & $85: 15$ & 6.9 & 35 \\
\hline & & $\mathrm{LH}$ & $93: 7$ & 6.5 & 36 \\
\hline Manzana & Fruto & $\mathrm{LH}$ & 13-mayoritaric & 6.0 & 37 \\
\hline \multirow[t]{2}{*}{ Patata } & Tubérculo & $\mathrm{LH}$ & $95: 5$ & 5.5 & 38 \\
\hline & & $\mathrm{LnH}$ & $95: 5$ & 5.5 & 38 \\
\hline Pera & Fruto & $\mathrm{LH}$ & $31: 69$ & $5.8-6.2$ & 3.9 \\
\hline \multirow[t]{3}{*}{ Soja } & Semilla & LH & $30: 70$ & 9.0 & 40 \\
\hline & & $\mathrm{LH}$ & $10: 90$ & 9.0 & 41 \\
\hline & & $\mathrm{LH}$ & $52: 48$ & 7.0 & 41 \\
\hline Tomate & Fruto & $\mathrm{LH}$ & $100: 0$ & 6.8 & 42 \\
\hline Trigo & Semilla & $\mathrm{LnH}$ & $100: 0$ & - & 43 \\
\hline
\end{tabular}


lipoxigenasa ha sido más ampliamente estudiada, se han identificado al menos 3 isoenzimas cuyas características están resumidas en la Tabla I. La isoenzima 1 se diferencia de las otras dos por su pH óptimo, que es alrededor de 9, y su baja actividad cooxidativa hacia carotenos. La diferencia entre la 2 y 3 está en una mayor actividad de la 2 hacia el-ácido araquidónico mientras la 3 es más activa con el ácido linoleico. La isoenzima 1 por su parte no muestra diferencia entre ambos sustratos (27). La estimulación con $\mathrm{Ca}^{2+}$ ocurre con la isoenzima 2 mientras que el $\mathrm{Ca}^{2+}$ inhibe la 3 . Una peculiaridad de la reacción aeróbica de la 3 es la formación de los oxodienos aparte de los correspondientes hidroperóxidos (27).

El mecanismo de acción de la lipoxigenasa, que se ha esquematizado en la Figura 3 empieza con la abstracción de un protón para formar un radical libre pentadienilo conjugado y posteriormente se produce la reacción del oxígeno con el mismo para dar lugar a los dos hidroperóxidos de la figura. Se ha comprobado (28) (29) que la acción catalítica es estereoespecífica produciéndose siempre un enantiómero determinado que depende del centro quiral que se crea. Así siempre se producen los 9- y 13-hidroperóxidos con configuración $R$ y $S$ respectivamente. Esto ha sido demostrado mediante el marcaje esteroespecífico con tritio de los hidrógenos de la posición 11, observándose la abstracción estereoespecífica de los mismos por un mecanismo que implica que el ataque del oxígeno se produce por la cara opuesta del plano que contiene el radical y de la cara donde se produce la abstracción del hidrógeno.

Los distintos hidroperóxidos se forman en diferentes proporciones dependiendo de la procedencia de la lipoxigenasa y del isoenzima utilizado. En la Tabla II se recogen algunos casos que muestran la diversidad de resultados dependiendo del origen del enzima. Como se puede observar, la mayoría de los estudios han sido realizados con el ácido linoleico, obteniéndose los dos posibles hidroperóxidos (9- y 13-) y, en algún caso, se observa la formación exclusiva de uno de ellos. Otros estudios han sido llevados a cabo con el ácido linolénico y se puede observar que también en estos casos los productos formados fueron el 9y el 13-hidroperóxidos.

Los diversos hidroperóxidos formados en las reacciones anteriormente descritas pueden sufrir una variedad de transformaciones tanto enzimáticas como no. Las transformaciones enzimáticas que sufren los hidroperóxidos lipídicos en alimentos vegetales serán discutidos en la parte 2 de esta revisión.

\section{AGRADECIMIENTO}

Este trabajo ha sido financiado en parte por la Comisión Interministerial de Ciencia y Tecnología
(Proyecto ALI 88-0169) y la Junta de Andalucía (Proyecto 2075). Los autores desean agradecer al Prof. Eduardo Vioque sus valiosas discusiones durante la realización del mismo.

\section{BIBLIOGRAFIA}

1. Gardner. H.W.,-"Oxidation of Lipids in Biological Tissue and Its Significance" en "Chemical Changes in Food during Processing". pp. 177-203.-Richardson, T. y Finley, J.M. (Eds). Avi Publishing Company, Inc. Westport, Conneticut, 1985.

2. Moncada, S., y Higgs, E.A.-"Metabolism of arachidonic acid".-Ann. N. Y. Acad. Sci 522 (1988) 454-463.

3. Shimizu, T.-"Enzymes functional in the synthesis of leukotrienes and related compounds".- Int. J. Biochem. 20 (1988) 661-666.

4. Irvine, R.F.- The role of phospholipids in the generation and action of mediators".-Res. Monogr. Cell Tissue Physiol. 15 (1988) 111 125. Chem. Abs. 109 (1988) 52742g.

5. Hammarstrom, S.-“Leukotrienes"- Ann. Rev. Biochem. 52 (1983) 355-377.

6. Hamberg, M., y Samuelsson, B.-"Prostaglandin endoperoxides. III. Novel transformations of arachidonic acid in human platelets",- Proc. Natl. Acad. Sci. USA 71 (1974) 3400-3404.

7. Borgeat, P.; Hamberg, M., y Samuelsson, B.-"Transformation of arachidonic acid and homo- $\gamma$-linolenic acid by rabbit polymorphonuclear leukocytes: Monohydroxy acids from novel lipoxygenase".- J. Biol. Chem. 251 (1976) 7816-7820.

8. Marcus, A.J.-"The role of lipids in platelet function with particular reference to the arachidonic acid pathway".- J. Lipid Res. 19 (1978) 793-826.

9. Zimmerman, D.C., y Coudron, C.A.-“Identification of traumatin, a wound hormone, as 12-oxo-trans-10-dodecenoic acid".- Plant Physiol. 63 (1979) 536-541.

10. Vick, B.A., y Zimmerman, D.C.-The biosynthesis of jasmonic acid: A physiological role for plant lipoxygenase".- Biochem. Biophys. Res. Commun. 111 (1983) 470-477.

11. McGill, A.S.; Hardy, R.; Burt, J.R., y Gunstone, F.D.-"Hept-cis4-enal and its contribution to the off-flavor in cold stored cod". $J$. Sci. Food Agric. 25 (1974) 1477-1489.

12. Swoboda, P.A.T., y Peers, K.E.-"Volatile odorous compounds responsible for metallic, fishy taint formed in butterfat by selective oxidation".-J. Sci. Food Agric. 28 (1977) 1010-1018.

13. Galliard, T.-"Degradation of acyl lipids: hydrolytic and oxidative enzymes" en "The Biochemistry of Plants: A Comprehensive Treatise. Vol. 4. Lipids: Structure and Function". pp 85-116.-Stumpf P.K. (Ed). Academic Press, New York, 1980.

14. Lin, Y.H.; Yu, C., y Huang, A.H.C.-"Substrate specificities of lipases from corn and other seeds".-Arch. Biochem. Biophys. 244 (1986) 346-356.

15. Huang, A.H.C.-"Lipases" en "The Biochemistry of Plants. A Comprehensive Treatise. Vol. 9. Lipids: Structure and Function", pp 91-120-Stumpf, P.K. y Conn, E.E. (Eds.). Academic Press, inc. New York, 1987.

16. Noma, A., y Borgstrom, B.-"Acid lipase of castor beans. Positional specificity and reaction mechanism".-Biochim. Biophys. Acta 227 (1971) 106-115.

17. Stumpf, P.K.-"Biosynthesis of Saturated and Unsaturated Fatty Acids" en "The Biochemistry of Plants: A Comprehensive Treatise. Vol. 4. Lipids: Structure and Function". pp. 177-204.-Stumpf, P.K. (Ed). Academic Press, New York, 1980.

18. Galliard, T.-"The enzymic deacylation of phospholipids and galactolipids in plants: purification and properties of a lipolitic acyl-hydrolase from potato tubers".-Biochem. J. 121 (1971) 379390. 
19. Hirayama, O.; Matsuda, H.; Takeda, H.; Maenaka, K., y Takatsuka, H.-"Purification and properties of a lipid acyl-hidrolase from potato tubers.-Biochim. Biophys Acta 384 (1975) 127-137.

20. Acker, L., y Beutler, H.O.-"Enzymatic fat hydrolysis in foodstuffs low in moisture".-Fette, Seifen, Anstrichm. 67 (1965) 430-433

21. Hatanaka, A.; Kajiwara, T., y Sekiya, J.-“Biosynthetic pathway for C6-aldehydes formation from linolenic acid in green leaves".-Chem Phys. Lipids 44 (1987) 341-361.

22. Pinsky, A.; Grossman S. y Trop. M.-“Lipoxygenase content and antioxidant activity of some fruits and vegetables".-J. Food Sci. 36 (1971) 571-572.

23. Douillard, R., y Bergeron, E.-"Lipoxygenase activity of wheat shoot chloroplasts".-C.R. Hebd. Seauces Acad. Sci. 286 (1978) 753755.

24. Grossman, S.; Ben Aziz, A.; Ascarelli, I., y Budowski, P.- "Intracellular distribution of lipoxygenase-like activity of alfalfa leaves".-Phytochemistry 11 (1972) 509-514.

25. Wardale, D.A., y Galliard, T.-"Further studies on the subcellular localization of lipid-degrading enzymes".-Phytochemistry 16 (1977) 333-338.

26. Haydar, M.; Steele, L., y Hadziyev, D.-"A study of lipoxidase in pea seed and seedlings".-J. Sci. Food Agric. 24 (1973) 10391053

27. Ramadoss, C.S., y Axelrod, B.-“High-performance liquid chromatografic separation of lipoxygenase isoenzymes in crude soybean extracts".-Anal. Biochem 127 (1982) 25-31.

28. Egmond, M.R.; Vliegenthart, J.F.G., y Boldingh, J.-“Stereospecificity of the hydrogen abstraction at carbon atom n-8 in the oxygenation of linoleic acid from corn germs and soya beans".-Biochem. Biophys. Res. Commun. 48 (1972) 1055-1060.

29. Hamberg, M., y Samuelsson, B.-"On the specificity of the oxygenation of unsaturated fatty acids catalyzed by soybean lipoxidase".-J. Biol. Chem. 242 (1967) 5329-5335.

30. Chang, C.C.; Esselman, W.J., y Clagett, C.O.-"Isolation and specificity of alfalfa lipoxygenase".-Lipids 6 (1971) 100-106.

31. Vioque, E., y Maza, M.P.-“'Oxidación del linoleato sódico por los extractos enzimáticos de algunas leguminosas".-Grasas y Aceites 22 (1971) 22-25.

32. Yamamoto, A.; Fujii, Y.; Yamamoto, K., y Mitsuda, H.- "Product specificity of rice germ lipoxygenase".-Lipids 15 (1980) 1-5.

33. Arens, D.; Seilmeilr, W.; Weber, F.; Kloos, G., y Grosch,
W.-"Purification and properties of a carotene co-oxidizing lipoxygenase from peas".-Biochim. Biophys. Acta 327 (1973) 295-305.

34. Zimmerman, D.C., y Vick, B.A.-"Specificity of flaxseed lipoxidase".-Lipids 5 (1970) 392-397.

35. Gardner, H.W., y Weisleder, D.-“Lipoxygenase from Zea mays: 9-D-hydroperoxy-trans-10-cis-12-octadecadienoic acid from linoleic acid".-Lipids 5 (1970) 678-683.

36. Hamberg, M.-“Steric analysis of hydroperoxides formed by lipoxygenase oxygenation of linoleic acid".-Anal. Biochem. 43 (1971) 515-526.

37. Kim, I.S., y Grosch, W.-"Partial purification of a lipoxygenase from apples".-J. Agric. Food Chem. 27 (1979) 243-246.

38. Galliard, T., y Phillips, D.R.-“Lipoxygenase from potato tubers: Partial purification and properties of an enzyme that specifically oxygenates the 9-position of linoleic acid".-Biochem. J. 124 (1971) $431-438$.

39. Kim, I.S., y Grosch, W.-“Lipoxygenases from pears, strawberries and gooseberries: partial purification and properties".- $Z$. Lebensm.-Unters. Forsch. 167 (1978) 324-326.

40. Veldink, G.A.; Vliengenthart, J.F.G.. y Boldingh, J.-"Proof of the enzymatic formation of 9-hydroperoxy-10-trans,12-cis-octadecadienoic acid by soya lipoxydase".-Biochim. Biophys. Acta 202 (1970) 198-199.

41. Christopher, J.P.; Pistorius, E.K.; Regnier, F.E., y Axelrod B.-"Factors influencing the positional specificity of soybean lipoxygenase".-Biochim. Biophys. Acta 289 (1972) 82-87.

42. Zamora, R.; Olias, J.M., y Mesias, J.L.-"Purification and characterization of tomato lipoxygenase".-Phytochemistry 26 (1987) 345347

43. Graveland. A.-"Enzymatic oxidation of linoleic acid in aqueous wheat flour suspensions".-Lipids 8 (1973) 606-611.

44. Galliard, T., y Chan, H.W.-S.-"Lipoxygenases" en "The Biochemistry of Plants: A Comprehensive Treatise. Lipids: Structure and Function" Vol. 4. pp. 131-161.-Stumpf, P.K. (Ed) Academic Press, New York, 1980.

(Recibido: Julio 1990) 